\title{
Numerical Simulation on the Characteristics of Refrigeration Air-Conditioning Heat Transfer Tube Based on the Current Situation of Subway Traffic
}

\author{
Weihua Ding \\ Guangzhou college, South China University of Technology, Guangzhou, Guangdong, 510800, China \\ dingxing1001@163.com
}

Keywords: Refrigerated Air-Conditioned Heat Transfer Pipe, Subway Traffic Status, Characteristic Numerical Simulation Experiment, Simulation Model.

\begin{abstract}
The present situation of subway traffic construction in big cities is very complicated, but in order to ensure the normal operation of the system, it is also necessary to do a good job of refrigeration and air conditioning system, in-depth control of refrigeration and air conditioning over temperature, and considering the asymmetry of the motor structure, a numerical simulation experimental system for the characteristics of refrigeration and air conditioning heat transfer pipe based on the current situation of subway traffic is established. In this paper, we will first establish a numerical simulation model of the characteristics of this refrigerated air-conditioning heat transfer tube, make relevant assumptions, establish a physical model around the solution domain, and finally establish a mathematical model to solve the boundary conditions, and experimentally verify and analyze the simulation results.
\end{abstract}

\section{Introduction}

Refrigeration and air conditioning heat transfer tube has a high status in subway traffic system, it realizes the effective regulation of temperature and humidity in subway space. However, because it is in the state of long-term operation without rest, the analysis of its heat transfer tube characteristics becomes very important. If the heat transfer tube overheating situation will inevitably lead to the refrigeration and air conditioning system motor internal overheating, insulation material damage, and finally form a short circuit, or even directly lead to the motor burning, so that the equipment into safety risks, the overall operation of the subway traffic system is also very unfavorable. From the technical point of view of the system, the over temperature will also bring the magnetic weakening of the magnetic material inside the motor, and even the demagnetization situation, so it is very necessary to explore the temperature field of the refrigeration air conditioning motor and its heat transfer tube.

\section{Overview of Refrigeration and Air-Conditioning System in Subway Traffic Situation}

The refrigeration and air conditioning system needs to run stably for a long time, but it is not easy to measure their actual temperature distribution in the process of real-time measurement because there are rotor parts in the motor of subway system, and the rotor part contains many kinds of rotating parts. At the same time, the internal temperature of the multi-solid parts in the motor is not easy to measure, and the requirements for measuring hardware and software are relatively high. Therefore, experts and scholars at home and abroad have deeply studied this problem, mainly for a part of the generator combined with stator, rotor and other local temperature field, flow field and other in-depth scientific research, the refrigeration and air conditioning system of the overall heat transfer characteristics of the motor, combined with the axial and circumferential symmetry analysis of the motor has been adjusted, using the axial 1/2, circumferential 1/6 symmetry model to carry out a detailed numerical simulation, specially for the stator coil blade, three-dimensional twist and other asymmetrical distribution characteristics of the analysis. The purpose of this paper is to study the specific temperature distribution of the motor in depth, and to measure the temperature of the motor 
experimentally.[1].

\section{The Model Construction of Refrigeration Air-Conditioning System under the Current Situation of Subway Traffic}

The model construction of refrigeration and air conditioning system based on the current situation of subway traffic mainly includes the basic hypothesis, the physical model construction of the solution domain, the mathematical model construction, the boundary condition analysis, the experimental verification and the simulation result analysis.[2].

\subsection{Basic assumptions}

In order to simplify the calculation process, it is necessary to first build a model for the heat transfer tube and electricity mechanism in the refrigeration and air conditioning system to realize the model optimization. The specific operation includes the following five points:

It is assumed that the loss of the motor is uniformly distributed on the same component.

It is necessary to ignore the screws, bolts, gaskets and other parts on the motor to ensure the heat transfer characteristics and flow characteristics of the heat transfer tube motor, and to minimize the influence of the flow characteristics of the secondary parts.

Third, it is necessary to match the structure of ball-milled cast iron, steel plate, carbon steel plate and so on in the motor with the pure iron material, mainly according to the physical properties of pure iron.

Fourth, we should study the physical properties of air in the motor and think about the characteristics of its temperature linear change, but at the same time, we should pay attention to the thermal conductivity of its heat transfer tube material, and look at the actual change process with the temperature.

Fifth, it is necessary to assume that the motor is always in a stable operation state, and make a targeted analysis of the continuous and stable working state of the air[3].

\subsection{Physical Modeling of Solution Domain}

Combined with the above 5-point analysis to solve the domain physical model, reasonable simplification of the motor three-dimensional model longitudinal section view content, as shown in figure 1.

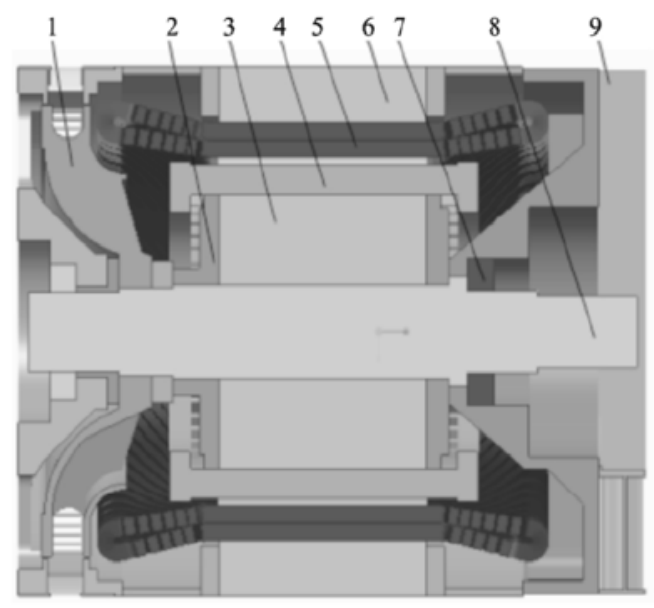

Figure 1 Three-dimensional model of heat transfer tube motor in air-conditioned refrigeration system

As shown in figure 1, there is a special heat transfer tube motor structure in the self-ventilated air conditioning refrigeration system, from 1 to 9 are fan, rotor press plate, rotor core, rotor coil, stator coil, stator core, bearing, rotary shaft, inlet filter dust device, respectively. its stator and rotor slots number are 60 and 48, where the stator has $165.0 \mathrm{~mm}$ and $255.0 \mathrm{~mm}$ inside and outside diameter, while the rotor has $165.0 \mathrm{~mm}$ and $255.0 \mathrm{~mm}$ inside and outside diameter, respectively. In rated 
state, the motor is able to operate stably, but it will cause iron loss, copper loss and various kinds of stray mechanical loss. At this time, it is necessary to use the fluid domain in the physical model of the solution domain to adjust the multiple rotation coordinates, to adjust the fluid domain, and then to solve the specific values of various losses $(\mathrm{kW})$. Taking the stator core as an example, if its total loss is about $4.33 \mathrm{~kW}$, then its volume loss is about $142028.1 \mathrm{~W} \cdot \mathrm{m}-3$. The solution domain model of heat transfer tube motor in subway refrigeration air conditioning system is shown in Fig .2.[4].

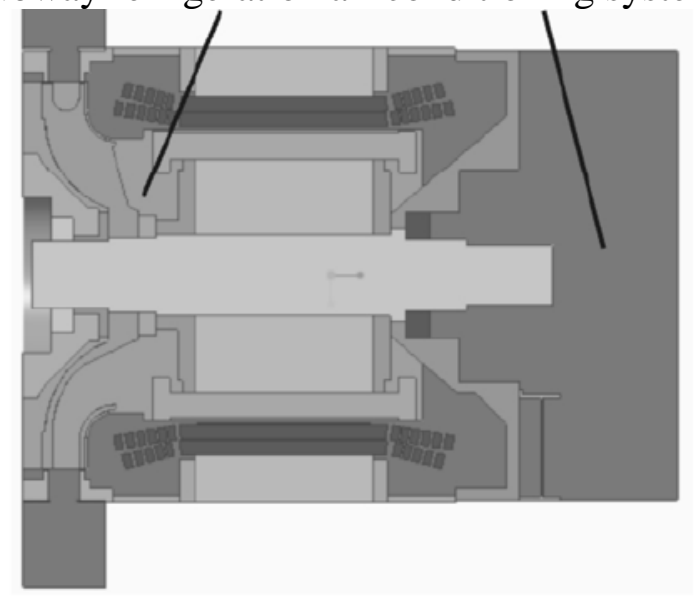

Figure 2 Schematic diagram of the solution domain model

When the heat transfer motor is in a high-speed rotating state, a certain range of low-voltage areas will be generated around the fan in the motor. In the pressure differential driving state, the low temperature air will pass through the inlet dust filter device to filter out the dust particles and enter the motor. At this time, it needs to be analyzed by the gas gap between the stator and the rotor, and more than 10 ventilation holes are evenly distributed to cool the rotor and stator of the motor, and the hot air is discharged from the fan radial grid at the same time.

\subsection{Mathematical Modelling}

Combining the above basic assumptions, the mathematical model is analyzed to ensure that the air is always in a continuous and stable working state, while ensuring that the air meets the continuity equation as follows:

$$
\operatorname{div}(\rho U)=0
$$

$U$ In the equation, it represents the air velocity. For the steady state of the subway traffic system, the turbulent momentum equation and the energy equation should be used, and then the standard equation should be written in the following general form: $k-\varepsilon$

$$
\operatorname{div}(\rho V \varphi)=\operatorname{div}(\operatorname{\Gamma grad} \varphi)+S
$$

$\varphi u 、 v 、 w x, y, z$ In the above formula, it represents a variety of variables and a velocity component in different axial directions in the air, corresponding to the generalized diffusion coefficient and the source coefficient, which can be calculated by using the standard turbulence model, which can be effectively treated with the standard wall function $k-\varepsilon$ [5].

\subsection{Analysis of Boundary Conditions}

In the process of boundary condition analysis, it is necessary to discuss the self-ventilation design of the heat transfer tube motor, and analyze the air flow rate of the inlet and outlet position through the rotational speed of the parts. The pressure inlet boundary condition can be set specifically for the inlet position to ensure that the relative pressure is 0 , and the boundary condition of no sliding wall at the interface between fluid and solid can be established by using interior boundary analysis.

$$
u_{\text {air }}=u_{\text {wall }}
$$


A third type of boundary condition is set up on the outer surface of the motor, a special static component is established based on the stator core, and the correlation formula is established according to the natural convection characteristic number of the horizontal cylinder in the air. Finally, the natural convection heat transfer coefficient of the static component in the refrigeration and air conditioning system is obtained. In general, the value range of the gas forced convection heat transfer coefficient should be controlled in the range of $20 \sim 100 \mathrm{~W} / \mathrm{m} 2 \cdot \mathrm{K}$ to ensure that the convection heat transfer coefficient is around $80 \mathrm{~W} / \mathrm{m} 2 \cdot \mathrm{K}$.

Finally, its temperature boundary condition is calculated, that is, the convenient condition of heat coupling between the flow-solid interface and the solid-solid interface is analyzed. The axial sheet structure air separation structure can be set at the outlet side fluid domain position of the system, and the calculation process can be simplified in modeling. Even the sheet structure can be neglected. the axial approximate adiabatic boundary content can be calculated directly at the outlet of the fluid domain to ensure its heat flux $=0 . q$

\subsection{Experimental Validation}

In order to further check the characteristics of refrigerated air conditioning heat transfer pipe in subway traffic, it is also necessary to carry out simulation experiments to measure the rated steady state operation of its motor. In the experiment, the embedded thermistor measurement method can be adopted, and the static sinusoidal power supply can be used in combination with the experimental power supply, and then the rotor high speed rotation analysis can be carried out to measure its temperature in real time, and the simulation effect can be obtained. In general, the simulation results show that the rotor temperature is relatively low compared to the stator, but the rotor part will not be overheated. the rated operating conditions of the motor during the experimental process are analyzed in combination with the experimental related parameters. the rated rotational speed is generally about $2000-2050 \mathrm{r} / \mathrm{min}$, and the steady state of the temperature field can be entered after about $250 \mathrm{~min}$ of operation. in this state, the temperature change of the temperature field in the period of $1 \mathrm{~h}$ is generally less than $1^{\circ} \mathrm{C}$, which can be approximated as the central plane temperature. Finally, according to the statistical results, the simulation calculation temperature results of the coil in the axial center section are analyzed, the simulation results are compared with the experimental results, the relative error problem is calculated, and the numerical simulation check results are obtained. In general, the simulation temperature of the coil slot is about $130 \sim 150^{\circ} \mathrm{C}$, and the relative error can be controlled in the range of $2.9 \sim 80 \%$, which basically meets the engineering requirements, which also verifies the importance of the numerical simulation for the heat transfer tube of the subway refrigeration air conditioning, and the simulation results are safe and reliable. [6].

\subsection{Analysis of Simulation Results}

As can be seen from the above experimental results, the temperature rise is effective for the temperature measurement of the stator coil in the refrigeration air-conditioning system. After analyzing the effect of the motor speed on the maximum temperature and the maximum temperature of the stator coil, it can be found that the maximum temperature rise and the maximum temperature of the stator coil are linearly changing with the increase of the system speed. inflow. when the rotational speed increases by about $1000 \mathrm{r} / \mathrm{min}$, the maximum temperature rise of the stator coil will rise by $6.5 \%$. As the speed of the motor increases gradually, the air flow rate continues to increase, and the cooling capacity is enhanced, and the temperature of the stator coil naturally increases with the increase of the speed. 


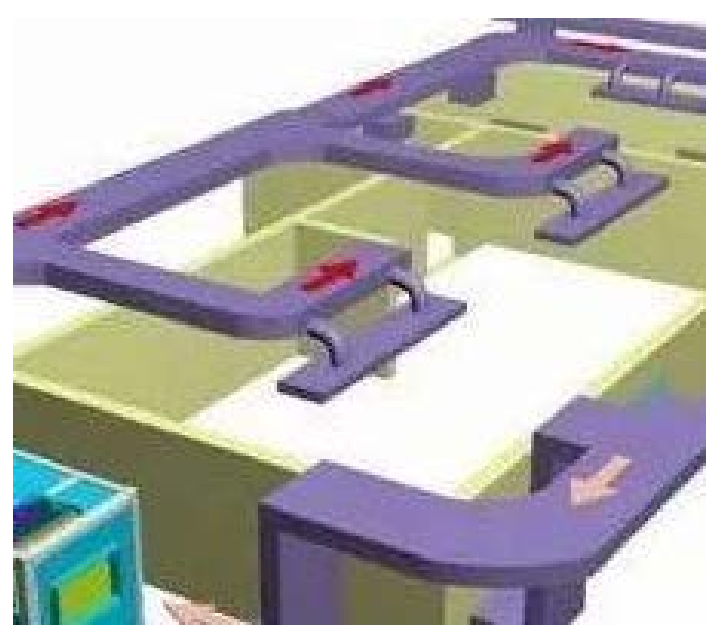

Figure 3 Simulation

\section{Conclusion}

Under the background of subway traffic, it is very necessary to simulate the characteristics of the heat transfer tube for the subway refrigeration air conditioning. It can analyze the flow heat transfer characteristics of the heat transfer tube under the rated working state, carry out the numerical analysis adjustment, measure the contents of the temperature field experimentally, explore the influence of the ambient temperature and the speed flow heat transfer characteristics, and obtain the final conclusion to prove all the views of this paper.

\section{References}

[1] Liang, Xueqi., Luo, Huiqiang., Wu, Yining. Numerical simulation and experimental study on heat transfer characteristics of subway motor. Journal of Xi'an Jiaotong University, vol. 52, no. 9, pp. 148-154, 2018.

[2] Zhou, Yang. Study on energy consumption of air conditioning system in summer during ground operation of subway vehicles. Sichuan: Southwest Jiaotong University, 2015.

[3] Qu, Kaiyang., Cao, Yang., Guo, Xu. iplv calculation method for water-cooled direct refrigeration air conditioning units in subway stations. Building Energy Saving, vol. 47, no. 11, pp. 52-57, 2019.

[4] Li, Feng., Fang, Long., Yang, Xinze. Performance test of direct cooling air conditioning units in subway station. HVAC, vol. 49, no. 12, pp. 115-119, 60, 2019.

[5] Chang, Xinyuan. Discussion on energy saving design of air conditioning refrigeration system for subway vehicles. China Science and Technology Investment, no. 10, pp. 184, 2109.

[6] Jia, Yingwu., Yin, Caihong. Subway air conditioning unit cooling auxiliary method research. Technology and Markets, vol. 26, no. 5, pp. 49-51, 2019. 